\title{
Mechanical Control of a Microrod-Resonator Optical Frequency Comb
}

\author{
Scott B. Papp, ${ }^{*}$ Pascal Del'Haye, and Scott A. Diddams \\ National Institute of Standards and Technology, Boulder, Colorado 80305, USA
}

(Received 27 February 2013; published 8 July 2013)

\begin{abstract}
We report on the stabilization of a microresonator-based optical frequency comb (microcomb) by way of mechanical actuation. These experiments use novel $\mathrm{CO}_{2}$-laser-machined microrod resonators, which are introduced here and feature optical $Q \geqslant 5 \times 10^{8}$, less than 1 minute processing time, and tunable geometry. Residual fluctuations of our $32.6 \mathrm{GHz}$ microcomb line spacing reach a stability level of $5 \times 10^{-15}$ for $1 \mathrm{~s}$ averaging, thereby highlighting the potential of microcombs to support modern opticalfrequency standards. Furthermore, measurements of the line spacing with respect to an independent frequency reference reveal stabilization of different spectral slices of the comb with a $<0.5-\mathrm{mHz}$ variation among $140 \mathrm{comb}$ lines spanning $4.5 \mathrm{THz}$. Together, these results demonstrate an important step in the development of microcombs, namely, that they can be fabricated and precisely controlled with simple and accessible techniques.
\end{abstract}

DOI: 10.1103/PhysRevX.3.031003

Control and stabilization of optical frequency combs enables a range of scientific and technological applications, including frequency metrology at high precision [1-3], spectroscopy of quantum gases [4] and of molecules from visible wavelengths to the far infrared [5], searches for exoplanets [6,7], and photonic waveform synthesis $[8,9]$. Recently, a new class of frequency combs based on monolithic microresonators has emerged, which offer significantly reduced bulk, cost, and complexity beyond what is possible with conventional femtosecond-laser technology [10]. Such factors stand in the way of nextgeneration applications that will require high-performance optical clocks for experiments outside the lab.

In microcomb systems, the comb generation relies on parametric conversion provided by third-order nonlinear optical effects and is enabled by high-quality factors $Q$ and small mode volumes of microresonators. To date, microcombs have been explored with a number of microresonator technologies, including microtoroids [11], crystalline resonators [12-14], microrings [15,16], fiber cavities [17], machined disks [18], and disk resonators [19]. Unique comb spectra have been demonstrated, featuring octave spans [20,21] and a wide range of line spacings [22]. And some aspects of the microcomb frequency-domain behavior have been explained [12,18,23-27].

Microcombs present an interesting challenge for frequency stabilization, as first pointed out in Ref. [24]. Specifically, the center frequency of a microcomb spectrum is matched to the pump laser, and the line spacing must be controlled by changing the resonator's physical

\footnotetext{
*scott.papp@nist.gov

Published by the American Physical Society under the terms of the Creative Commons Attribution 3.0 License. Further distribution of this work must maintain attribution to the author(s) and the published article's title, journal citation, and DOI.
}

Subject Areas: Optics, Optoelectronics, Photonics

properties. Future metrology applications of microcombs will require stabilization of the line spacing with respect to fixed-optical and microwave frequency standards. Hence, the key factors for stabilization are a line spacing in the measurable 10's of $\mathrm{GHz}$ range (Ref. [28] describes a technique at $>100 \mathrm{GHz}$ ), low intrinsic fluctuations, and the capability for fast modulation. Additionally, a threshold power for comb generation in the milliwatt range and the potential for integration with chip-based photonic circuits would enable portable applications.

Here, we report a new microcomb platform for achieving these goals. We have developed a $\mathrm{CO}_{2}$-laser-machining technique that yields microrod optical resonators with a $Q \gtrsim 5 \times 10^{8}$, a user-defined diameter, and a small effective mode area. The optical modes of these cavities can be rapidly controlled by using mechanical forces that alter the resonator's shape $[29,30]$. With such microrods, we create a comb spectrum with 32.6-GHz-spaced lines spanning from $1510 \mathrm{~nm}$ to $1620 \mathrm{~nm}$. Our work introduces wideband mechanical control of the microcomb line spacing and its stabilization with respect to microwave standards. Mechanical control offers significant advantages, including low-power operation, simple integration with bulk resonators, and response potentially much faster than resonator thermal conduction [31]. We have improved the residual line-spacing fluctuations by more than a factor of 200 beyond previous microcomb work [24]. And we demonstrate the potential of microcombs to support optical frequency references that feature stability in the $10^{-15}$ range.

To create microrod resonators, we use a $\mathrm{CO}_{2}$ laser to simultaneously shape and polish a fused-quartz rod (Fig. 1). At a 10.6- $\mu \mathrm{m}$ wavelength, fused quartz is highly absorptive, such that melting and evaporation of the material is easily accomplished with a focused $\mathrm{CO}_{2}$ laser beam of less than $5 \mathrm{~W}$. Figure 1(a) illustrates the procedure for resonator fabrication. A 2-mm-diameter fused-quartz rod 

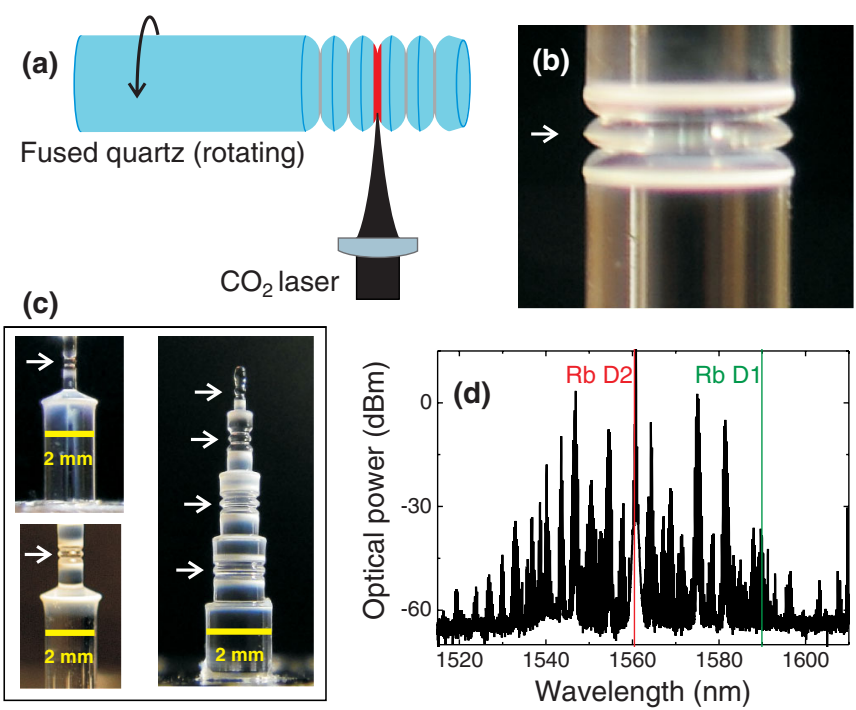

FIG. 1. Fabrication of fused-quartz microrod resonators by use of $\mathrm{CO}_{2}$-laser machining. (a) $\mathrm{A}$ rotating fused-quartz rod is illuminated with a focused $\mathrm{CO}_{2}$ laser that selectively removes material. By applying the laser at different positions along the rod's axis, a microresonator is produced. (b) Image of a microrod with $Q=5 \times 10^{8}, 2$-mm diameter, and approximately $100-\mu \mathrm{m}$ mode cross-sectional diameter. (c) Fabrication of resonators with variable diameter. Starting at top left and counting clockwise, the resonator diameters are $0.58 \mathrm{~mm}, 0.36 \mathrm{~mm}, 0.71 \mathrm{~mm}, 1.2 \mathrm{~mm}$, $1.5 \mathrm{~mm}$, and $1.0 \mathrm{~mm}$. (d) Optical spectrum from the device in (b), which has a modulated envelope characteristic of parametric combs $[14,18,36]$. The span of this comb is sufficient to access the D1 and D2 transitions of atomic Rb, following secondharmonic generation.

is rotated in a ball-bearing spindle, and the $\mathrm{CO}_{2}$ laser is directed normal to the axis of the rod. The basic shape of a spheroidal resonator [Fig. 1(b)] is created by iteratively applying 5-s laser pulses at locations laterally separated by $0.3 \mathrm{~mm}$. This process also limits redeposition of material on the resonator surface. The machining self-terminates when the volume of material subjected to the $\mathrm{CO}_{2}$ laser is removed. Microrod laser machining offers several unique features, including a $<1$-min fabrication time, built-in polishing of the resonator surface to support ultrahigh $Q$, and a resonator yield of nearly $100 \%$ with $Q$ factors around $5 \times 10^{8}$. A demonstration is included in Videos 1 and 2 and Ref. [32]. In comparison, our earlier technique of diamond grinding and gas-flame polishing [18] is significantly slower and less repeatable, and it requires more skilled handwork than our new $\mathrm{CO}_{2}$ technique.

We discovered that self-termination of the $\mathrm{CO}_{2}$ process enables control over the resonator diameter with \pm 10 - $\mu \mathrm{m}$ precision. Before machining a resonator, we can arbitrarily reduce the quartz-rod diameter by positioning the $\mathrm{CO}_{2}$ laser beam above (or below) the rod and repeatedly moving it back and forth along the rod's axis. All the quartz material subjected to sufficiently high laser power is removed. Moreover, this procedure dresses the rod with respect to

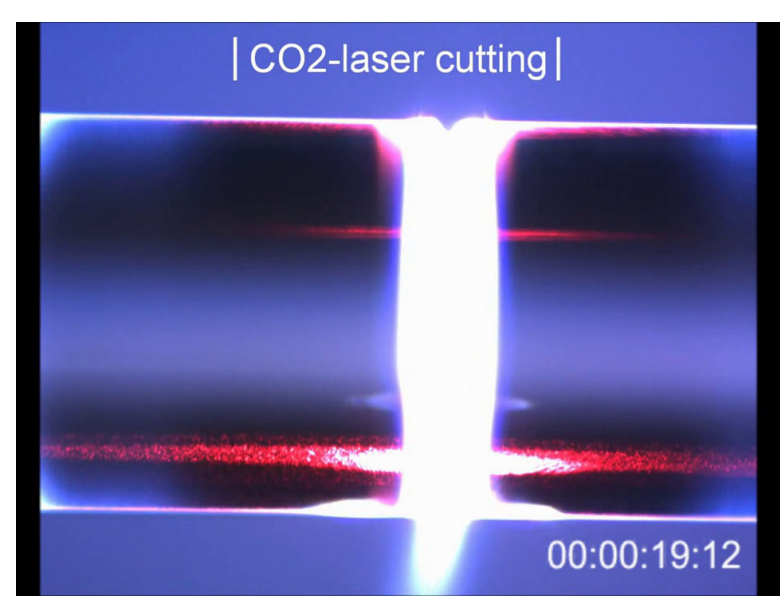

VIDEO 1. Video demonstration of microrod-resonator fabrication using $\mathrm{CO}_{2}$ laser machining. A 2-mm-diameter fusedquartz rod is rotated with a spindle, and it is shaped and polished by the laser. The laser beam translates between two axial positions along the rod by adjusting the focus lens. Total fabrication time is $<1 \mathrm{~min}$.

the fixed position of the laser beam. Figure 1(c) shows fused-quartz rods that were turned down in this manner. We have fabricated microrods that produce combs with line spacings from $8 \mathrm{GHz}$ to $380 \mathrm{GHz}$, highlighting the potential for nonlinear optics in these devices [33]. The image on the right in Fig. 1(c) demonstrates our capability to fabricate microrods of varying diameter on a single fused-quartz sample. This feature will enable future experiments that require control of the spacing between resonator modes.

To generate microcomb spectra [Fig. 1(d)], we pump a microrod with light coupled via a tapered fiber [34]. The

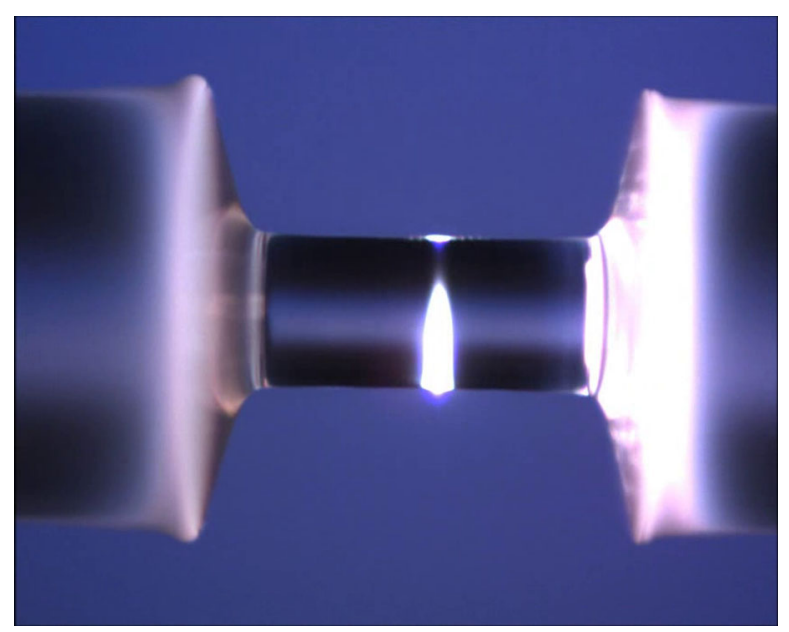

VIDEO 2. Video showing laser turning of a fused-quartz rod, followed by resonator fabrication. The laser beam is scanned along the axis of the rod for continuous material removal. This procedure enables wide control over the diameter of microrod resonators. Commentary during the video provides context. 
pump laser, a tunable semiconductor laser operating near $1560 \mathrm{~nm}$, is amplified in erbium fiber and then spectrally filtered to remove noise from amplified spontaneous emission; $280 \mathrm{~mW}$ of light is available at the input to the tapered fiber. The microrod is thermally self-locked to the pump laser [35], allowing us to stabilize the microcomb center to an auxiliary laser, which in turn is frequency doubled and referenced to a rubidium D2 transition at $780 \mathrm{~nm}$. The $\mathrm{Rb}$ atoms provide an absolute fractional stability of approximately $10^{-11}$ at $1 \mathrm{~s}$, but the 1 -s residual noise of $<10^{-17}$ between the microcomb pump and the auxiliary laser indicates that much more stable references can be employed in the future. The spectrum of our comb, which spans about $100 \mathrm{~nm}$, also reaches the corresponding wavelength $(1590 \mathrm{~nm})$ of Rb D1 lines at $795 \mathrm{~nm}$. This opens the possibility for all-optical stabilization of the comb center and line spacing using Rb transitions; a future paper will explore this idea. In this article, we focus on characterization and stabilization of the 32.6-GHz line spacing, which is measured by photodetection. After conversion to baseband (described below), the line-spacing signal is analyzed with respect to ultralow phase- and frequency-noise hydrogen-maser oscillators. We optimize the line spacing using resonator mode, taper-resonator coupling, and pump detuning and polarization, such that its spectrum exhibits only a single clean peak; see Ref. [32]. Under different conditions, particularly at high pump power, we observe multiple peaks in the line-spacing signal $[18,25]$. These effects prevent us from using $33-\mathrm{GHz}$ microrod combs with more continuous and smoother intensity envelopes, and bandwidths up to approximately $150 \mathrm{~nm}$. Figure 2(d) shows a two-hour record of the free-running microcomb line spacing, which is an important measure of comb performance. The 1-s Allan deviation for 100-s increments of these data, taken under typical laboratory conditions, ranges from $2 \times 10^{-8}$ to $2 \times 10^{-7}$.

Here, we introduce a mechanism for control of the comb's line-spacing noise via a mechanical force applied along the axis of the fused-quartz rod. An image of our setup for line-spacing control is shown in Fig. 2(a). A leadzirconate-titanate (PZT) element is used to compress the fused-quartz rod, resulting in radial expansion and tuning of the resonator's mode structure. In Figs. 2(b) and 2(c), we characterize the modulation response of a resonator mode and the line spacing of our comb, respectively. For a pump power well below thermal hysteresis, we monitor the resonance frequency of a mode as the PZT voltage is varied; see Fig. 2(b). The PZT adjusts the mode frequency by $5 \mathrm{MHz} / \mathrm{V}$ (approximately $800 \mathrm{MHz}$ total range) below a mechanical resonance of the system at $25 \mathrm{kHz}$. This response is less than what is expected $\left(P_{\mathrm{PZT}} \nu / E \times\right.$ $192 \mathrm{THz}=140 \mathrm{MHz} / \mathrm{V})$, given Young's modulus $E$ and Poisson ratio $\nu$ for fused quartz, and the approximately $0.3-\mathrm{MPa} / \mathrm{V}$ PZT stroke. The discrepancy is likely explained by a poor mechanical connection, but the lower
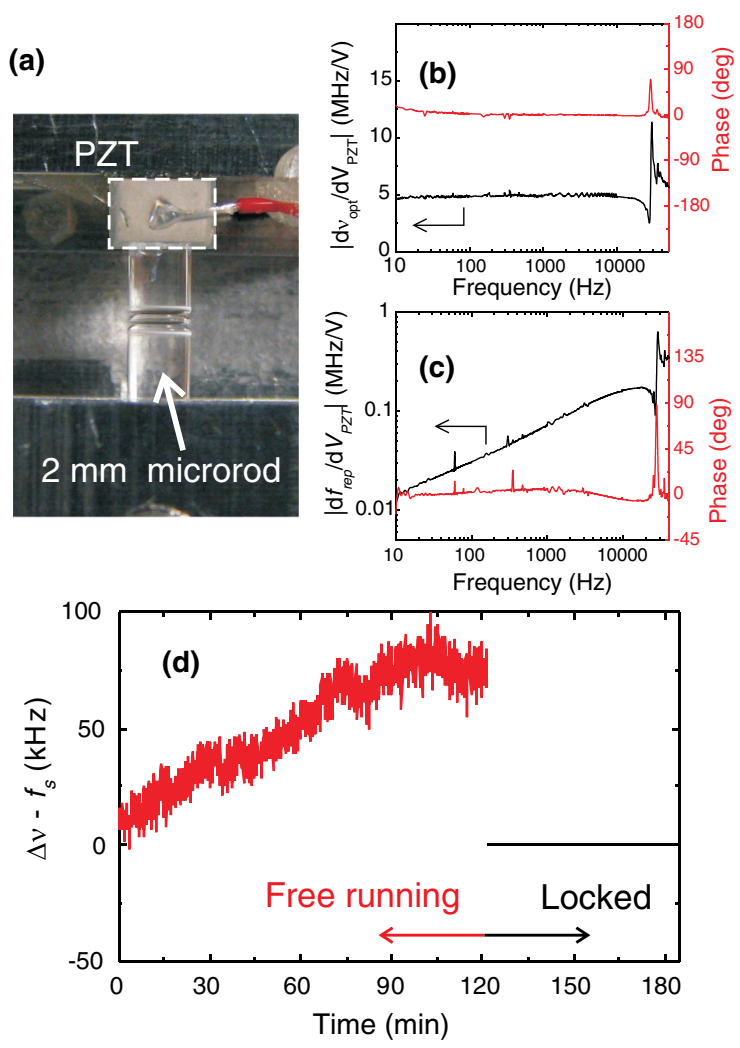

FIG. 2. Mechanical control mechanism for microresonator combs. (a) Control apparatus. A PZT compresses the microrod resonator to adjust its mode frequencies. (b, c) Response of optical resonance (b) and microcomb line spacing (c) with PZT drive frequency. (d) A three-hour record of the microcomb line spacing $(\Delta \nu)$ under free-running and stabilized conditions. The line-spacing lock point $f_{s}$ is $32.5671 \mathrm{GHz}$.

response has not limited our experiments. Finite-element simulations suggest that the off-center PZT-microrod mounting we use is not yet optimized for generating compression; see Ref. [32]. The line spacing of the comb also tunes with PZT voltage up to $25 \mathrm{kHz}$; however, resonator thermal locking reduces the response at low frequencies. A near-zero phase delay between the modulation and the PZT-induced response indicates the passive nature of the thermal lock, and it satisfies a basic requirement for providing useful feedback. The PZT enables stabilization of the line spacing, which is evident starting at $120 \mathrm{~min}$ in Fig. 2(d), for a wide range of pump-resonator detunings and for different resonator modes. Conversely, we also observe turning points in the PZT control, at which the line-spacing response (and noise) is reduced. This behavior is well known from laser-based combs.

We analyze the line spacing in detail to understand a microcomb's potential for replicating in each comb line the stability of state-of-the-art frequency references. Figure 3(a) shows the important elements of our apparatus. Following generation, the microcomb spectrum is delivered to two systems for independent stabilization 

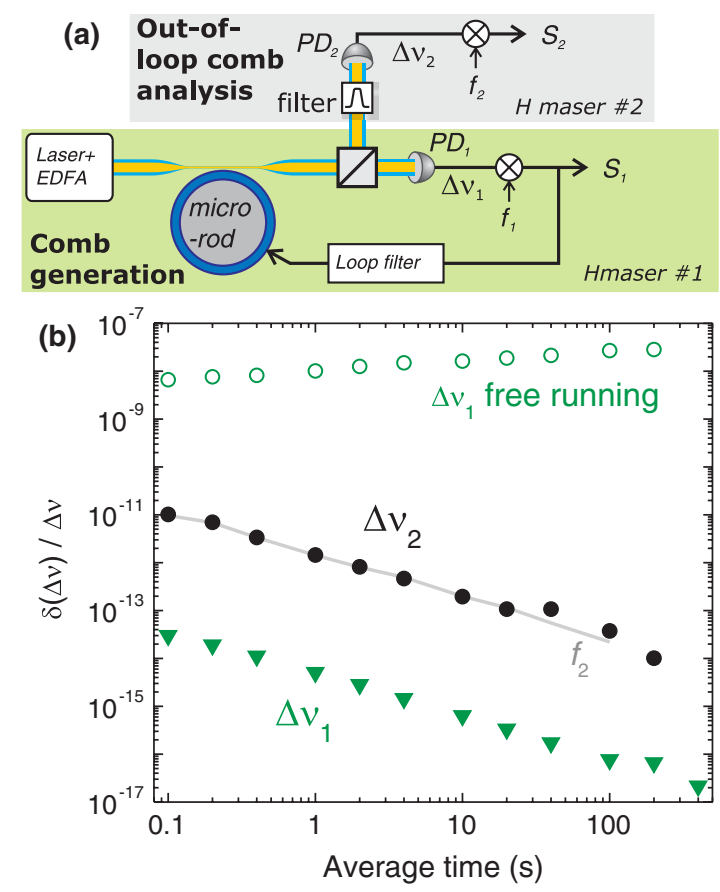

FIG. 3. Microcomb line-spacing stability. (a) Schematic of our systems for microcomb generation and line-spacing stabilization (green box), and "out-of-loop" analysis (gray box). The entire optical path is in fiber, including a programmable optical filter used to study the line-spacing stability for portions of the comb (Fig. 4). Commercial frequency synthesizers create references $f_{1,2}$ that are used for baseband conversion, and signals $S_{1,2}$ are measured. The generation and analysis systems have independent maser references. (b) Line-spacing Allan deviation versus averaging time for (triangles) stabilized residual, (points) stabilized absolute, and (open circles) free running. The gray line shows the Allan deviation of reference $f_{2}$.

and analysis. In both systems, the $32.6 \mathrm{GHz}$ comb line spacing $\left(\Delta \nu_{1}\right.$ and $\left.\Delta \nu_{2}\right)$ is photodetected, amplified, and converted to the baseband signals $S_{1}$ and $S_{2}$. Importantly, $S_{1,2}$ carry the fluctuations of both the line spacing and the microwave synthesizers $\left(f_{1}\right.$ and $\left.f_{2}\right)$, which are locked to independent maser signals. Amplifier noise is less important than that of the synthesizers. The Allan deviation and phase-noise spectra of signals $S_{1,2}$ are recorded separately with a Symmetricom 5125A phase-noise and Allandeviation test set, which is referenced to maser 1 (2) for $S_{1}\left(S_{2}\right)$ measurements. By initiating a phase-locked loop using $S_{1}$ and the PZT, we stabilize $\Delta \nu_{1}$ with respect to maser 1 . At an averaging time of $1 \mathrm{~s}$, the $5 \times 10^{-15}$ residual fluctuations of $\Delta \nu_{1}$ [green triangles in Fig. 3(b)] are far below the stability of maser 1 . This result signifies that the microcomb closely follows the reference frequency $f_{1}$ and attains its stability. Our analysis system (referenced to maser 2) tests the microcomb's ability to characterize independent microwave frequencies such as $f_{2}$. In Fig. 3(b), the filled points show the combined fluctuations of $\Delta \nu_{2}$ and $f_{2}$, which are predominately due to $f_{2}$; the solid line in Fig. 3(b) shows the Allan deviation of $f_{2}$ from a separate measurement. These data confirm the expectation from our residual measurements that the absolute stability of $\Delta \nu_{2}$ is significantly better than the $f_{2}$-synthesizer-limited $1.5 \times 10^{-12}$ at $1 \mathrm{~s}$. The consistent $1 /$ time averaging behavior observed in both our residual and absolute measurements is evidence of the phaselocked stabilization. In contrast, the open circles in Fig. 3(b) show the free-running line spacing.

The $S_{1}$ signal used for line-spacing stabilization is a composite of all the comb lines, and its largest contributions naturally come from the most intense pairs. Hence, an uneven distribution of comb optical power, along with the complicated nonlinear comb-generation process, opens the possibility of degraded line-spacing stabilization for different spectral slices of the comb. To quantify these effects, we probe the line-spacing frequency and its stability with our (maser-2 referenced) analysis system. A $1535-\mathrm{nm}$ to $1565-\mathrm{nm}$ (C-band) programmable optical filter with $10 \mathrm{GHz}$ resolution is used ["filter" in Fig. 3(a)] to select a portion of the comb (Fig. 4(a)) for $\Delta \nu_{2}$ analysis. Figure 4(b) shows measurements of the difference in line spacing $\left(\Delta \nu_{1}-\Delta \nu_{2}\right)$ between the entire comb and various portions of it. Here, the horizontal bars correspond to the transmission window of the filter for each $\Delta \nu_{2}$ measurement, and $\Delta \nu_{1}$ is determined by the lock point of our phase-locked loop. (The residual offset between maser 1 and locked $S_{1}$ is $<1 \mu \mathrm{Hz}$.) The weighted mean of all data is $-0.4 \mathrm{mHz}$ (on the 32.6-GHz line spacing) from the anticipated null, which is consistent with our measurement uncertainty. For these measurements, we subtract 21.3(13) $\mathrm{mHz}$, which arises from the frequency offset between the two independent masers that reference $f_{1}$ and $f_{2}$. A fit of the slope in Fig. 4(b) demonstrates that the line spacing does not change by more than the $5 \times 10^{-15}$ standard error over a $4.5-\mathrm{THz}$ span of the comb.

The line-spacing stability of the spectral slices also characterizes the PZT stabilization. Figure 4(c) shows the 1-s Allan deviation associated with each 400-s long frequency difference measurement. The stability of $\Delta \nu_{2}$ throughout the $C$-band portion of the comb is $1.5 \times$ $10^{-12}$, a value dominated by frequency synthesizer $f_{2}$. It appears that the mechanisms responsible for line-spacing noise act similarly to different components of the comb, and our PZT control can effectively counter them. To understand the residual stability of $\Delta \nu_{2}$ that is possible apart from the noise of $f_{2}$, we reconfigure our system to use $f_{1}$ for baseband conversion of both $\Delta \nu_{1}$ and the optically filtered $\Delta \nu_{2}$. In this case, common $f_{1}$ noise contributions are suppressed when the $S_{1,2}$ signals are presented to our noise analyzer. What remains are uncontrolled jitter between the spectral slices and the whole comb, and the noise associated with the independent optical and electrical measurement paths including the Symmetricom analyzer. The 


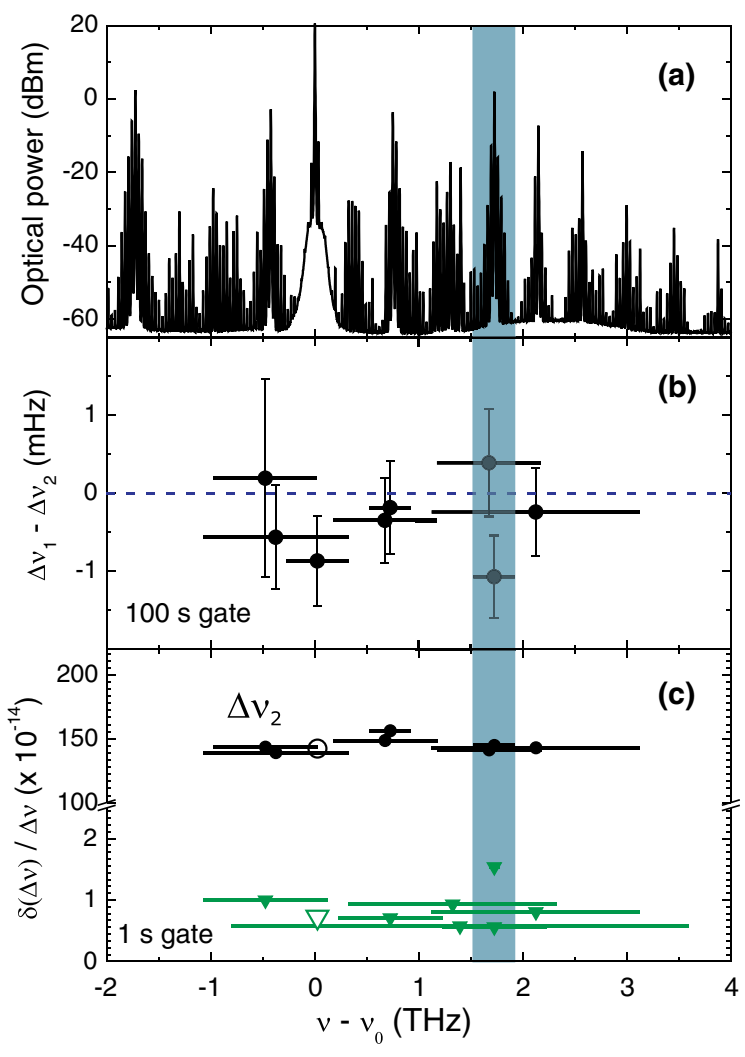

FIG. 4. Line-spacing equidistance and stability for different spectral slices of the comb. (a) Microcomb optical spectrum about the pump laser frequency $\nu_{0}$ prior to filtering. (b) Measurements of the frequency difference $\left(\Delta \nu_{1}-\Delta \nu_{2}\right)$ between the whole comb and a spectral slice. The horizontal bars indicate the frequency range of each measurement, which is highlighted by the shaded region for one point. (c) For different spectral slices, the points show the 1-s Allan deviation of $\Delta \nu_{2}$ measured against maser 2 , and the triangles characterize residual fluctuations between $\Delta \nu_{1}$ and $\Delta \nu_{2}$ measured against maser 1. For the open circle and open triangle data points, only a $0.3-\mathrm{THz}$ range about $\nu_{0}$ is blocked.

level of these residual fluctuations is mostly below $10^{-14}$ at $1 \mathrm{~s}$; see the green triangles in Fig. 4(c). This demonstrates that future microcomb experiments could take advantage of frequency references even more stable than a maser.

To understand the pathway for future improvements in line-spacing stability, we characterize the free-running noise spectrum of $\Delta \nu_{1}$; see the black curve in Fig. 5. Our servo electronics reduce the frequency-noise spectrum by up to $10^{5}$ within the $25-\mathrm{kHz}$ bandwidth permitted by the PZT, and the spectrum after stabilization is shown by the green curve in Fig. 5. Achieving further reduction in $S_{\Delta \nu_{1}}$ will depend on improvements among the feedback mechanism and the underlying source of the noise. Here, we focus on the latter. In our current system, the primary contribution to $S_{\Delta \nu_{1}}$ is pump-frequency noise that maps onto the line spacing via a mostly constant relationship $\gamma_{f}=10 \mathrm{~Hz}_{\Delta \nu_{1}} / \mathrm{kHz}$. This calibration was performed by modulating the pump frequency and recording the

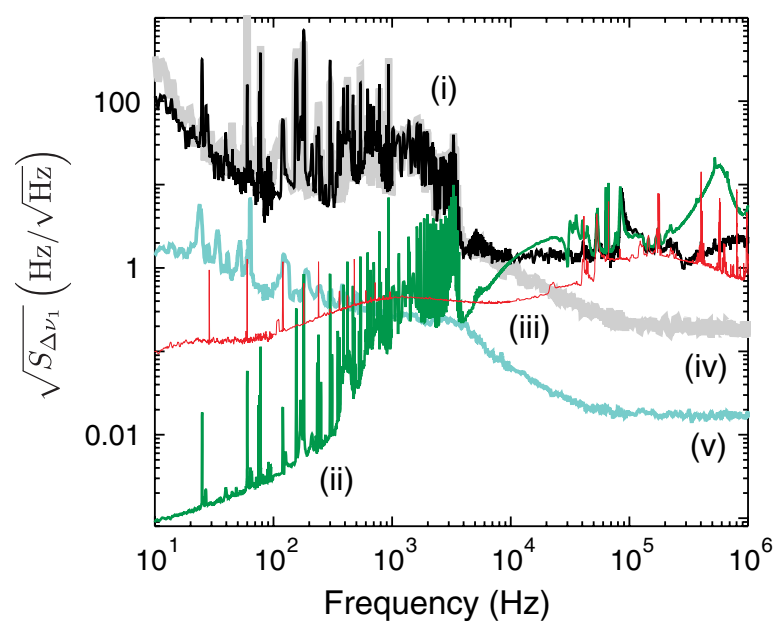

FIG. 5. Spectrum of line-spacing fluctuations $S_{\Delta \nu_{1}}$. The black (i) and green (ii) lines show the free-running and stabilized line-spacing spectral density, respectively. The broad resonance at $600 \mathrm{kHz}$ in the green line coincides with a mechanical resonance of the fused-quartz rod, which we speculate is weakly excited via the PZT. The red (iii) line indicates the contribution from reference $f_{1}$. The gray (iv) and blue (v) lines show the predicted contributions from pump frequency and intensity noise, respectively.

associated modulation in $\Delta \nu_{1}$. By measuring the spectral density of pump-frequency noise and scaling it by $\gamma_{f}$, we obtain the gray curve in Fig. 5. We also characterize the degree that pump intensity noise contributes to $S_{\Delta \nu_{1}}$. In this case, the mapping relationship is $\gamma_{P}=1.9 \mathrm{kHz} / \mathrm{mW}$, and it leads to the blue curve in Fig. 5. It is surprising that intensity noise does not contribute more significantly to $S_{\Delta \nu_{1}}$, especially in light of previous data [18]. Still, our characterization of $S_{\Delta \nu_{1}}$ suggests that a lower noise pump laser should be used in future experiments. In particular, with a factor of 10 improvement, a residual frequency noise of $<100 \mu \mathrm{Hz} / \sqrt{\mathrm{Hz}}$ at a $10-\mathrm{Hz}$ offset from the 32.6-GHz line-spacing signal would be possible. Noise contributions from our measurement system also appear in $S_{\Delta \nu_{1}}$. The red line shows the spectrum of $f_{1}$, which is generated by a high-performance commercial synthesizer.

In conclusion, we have introduced new techniques for fabricating microresonators with $Q \geqslant 5 \times 10^{8}$ and for controlling the line spacing of frequency combs created with them. These resonators are simple to create, and we have presented a deterministic procedure for varying their diameter. Furthermore, we have reported a detailed study of microcomb line-spacing stabilization using piezoelectric mechanical control. This type of mechanical line-spacing control can be introduced into a variety of microcomb generators based on, for example, crystalline resonators or chip-integrated devices via bending of the chip. Our work has demonstrated microcomb residual noise that is capable of supporting modern frequency references beyond the $10^{-13}$ at 1 -s level associated with the best traditional 
microwave oscillator technology. Future work will focus on increasing the frequency span of the comb and on reducing instabilities in the comb spectrum associated with competing parametric generation processes [25].

We thank Chris Oates, Kerry Vahala, and Gabe Ycas for their comments on this manuscript. This work is supported by the DARPA QuASAR program, NASA, and NIST. This paper is a contribution of NIST and is not subject to copyright in the United States. Mention of specific trade names does not constitute an endorsement. S.P. acknowledges support from the U.S. National Research Council, and P. D. thanks the Humboldt Foundation for support.

[1] S. A. Diddams, J. C. Bergquist, S. R. Jeerts, and C. W. Oates, Standards of Time and Frequency at the Outset of the 21st Century, Science 306, 1318 (2004).

[2] T. Rosenband, D. B. Hume, P. O. Schmidt, C. W. Chou, A. Brusch, L. Lorini, W. H. Oskay, R. E. Drullinger, T. M. Fortier, J. E. Stalnaker, S. A. Diddams, W. C. Swann, N. R. Newbury, W. M. Itano, D. J. Wineland, and J. C. Bergquist, Frequency Ratio of $\mathrm{Al}^{+}$and $\mathrm{Hg}^{+}$Single-Ion Optical Clocks; Metrology at the 17th Decimal Place, Science 319, 1808 (2008).

[3] Y. Y. Jiang, A. D. Ludlow, N. D. Lemke, R. W. Fox, J. A. Sherman, L.-S. Ma, and C. W. Oates, Making Optical Atomic Clocks More Stable with $10^{-16}$-Level Laser Stabilization, Nat. Photonics 5, 158 (2011).

[4] K.-K. Ni, S. Ospelkaus, M. H. G. de Miranda, A. Pe'er, B. Neyenhuis, J. J. Zirbel, S. Kotochigova, P. S. Julienne, D. S. Jin, and J. Ye, A High Phase-Space-Density Gas of Polar Molecules, Science 322, 231 (2008).

[5] Scott A. Diddams, Leo Hollberg, and Vela Mbele, Molecular Fingerprinting with the Resolved Modes of a Femtosecond Laser Frequency Comb, Nature (London) 445, 627 (2007).

[6] Tilo Steinmetz, Tobias Wilken, Constanza Araujo- Hauck, Ronald Holzwarth, Theodor W. Hansch, Luca Pasquini, Antonio Manescau, Sandro D’Odorico, Michael T. Murphy, Thomas Kentischer, Wolfgang Schmidt, and Thomas Udem, Laser Frequency Combs for Astronomical Observations, Science 321, 1335 (2008).

[7] Gabriel G. Ycas, Franklyn Quinlan, Scott A. Diddams, Steve Osterman, Suvrath Mahadevan, Stephen Redman, Ryan Terrien, Lawrence Ramsey, Chad F. Bender, Brandon Botzer, and Steinn Sigurdsson, Demonstration of onSky Calibration of Astronomical Spectra Using a $25 \mathrm{GHz}$ Near-IR Laser Frequency Comb, Opt. Express 20, 6631 (2012).

[8] Zhi Jiang, Chen-Bin Huang, Daniel E. Leaird, and Andrew M. Weiner, Optical Arbitrary Waveform Processing of More than 100 Spectral Comb Lines, Nat. Photonics 1, 463 (2007).

[9] T. M. Fortier, M. S. Kirchner, F. Quinlan, J. Taylor, J. C. Bergquist, T. Rosenband, N. Lemke, A. Ludlow, Y. Jiang, C. W. Oates, and S. A. Diddams, Generation of Ultrastable Microwaves via Optical Frequency Division, Nat. Photonics 5, 425 (2011).
[10] T. J. Kippenberg, R. Holzwarth, and S. A. Diddams, Microresonator-Based Optical Frequency Combs, Science 332, 555 (2011).

[11] P. Del'Haye, A. Schliesser, O. Arcizet, T. Wilken, R. Holzwarth, and T.J. Kippenberg, Optical Frequency Comb Generation from a Monolithic Microresonator, Nature (London) 450, 1214 (2007).

[12] Anatoliy A. Savchenkov, Andrey B. Matsko, Vladimir S. Ilchenko, Iouri Solomatine, David Seidel, and Lute Maleki, Tunable Optical Frequency Comb with a Crystalline Whispering Gallery Mode Resonator, Phys. Rev. Lett. 101, 093902 (2008).

[13] Ivan S. Grudinin, Nan Yu, and Lute Maleki, Generation of Optical Frequency Combs with a $\mathrm{CaF}_{2}$ Resonator, Opt. Lett. 34, 878 (2009).

[14] Yanne K. Chembo, Dmitry V. Strekalov, and Nan Yu, Spectrum and Dynamics of Optical Frequency Combs Generated with Monolithic Whispering Gallery Mode Resonators, Phys. Rev. Lett. 104, 103902 (2010).

[15] Jacob S. Levy, Alexander Gondarenko, Mark A. Foster, Amy C. Turner-Foster, Alexander L. Gaeta, and Michal Lipson, CMOS-Compatible Multiple-Wavelength Oscillator for on-Chip Optical Interconnects, Nat. Photonics 4, 37 (2009).

[16] L. Razzari, D. Duchesne, M. Ferrera, R. Morandotti, S. Chu, B.E. Little, and D.J. Moss, CMOS-Compatible Integrated Optical Hyper-Parametric Oscillator, Nat. Photonics 4, 41 (2009).

[17] Danielle Braje, Leo Hollberg, and Scott Diddams, Brillouin-Enhanced Hyperparametric Generation of an Optical Frequency Comb in a Monolithic Highly Nonlinear Fiber Cavity Pumped by a cw Laser, Phys. Rev. Lett. 102, 193902 (2009).

[18] Scott B. Papp and Scott A. Diddams, Spectral and Temporal Characterization of a Fused-QuartzMicroresonator Optical Frequency Comb, Phys. Rev. A 84, 053833 (2011).

[19] Hansuek Lee, Tong Chen, Jiang Li, Ki Youl Yang, Seokmin Jeon, Oskar Painter, and Kerry J. Vahala, Chemically Etched Ultrahigh-Q Wedge-Resonator on a Silicon Chip, Nat. Photonics 6, 369 (2012).

[20] P. Del'Haye, T. Herr, E. Gavartin, M. L. Gorodetsky, R. Holzwarth, and T.J. Kippenberg, Octave Spanning Tunable Frequency Comb from a Microresonator, Phys. Rev. Lett. 107, 063901 (2011).

[21] Yoshitomo Okawachi, Kasturi Saha, Jacob S. Levy, Y. Henry Wen, Michal Lipson, and Alexander L. Gaeta, Octave-Spanning Frequency Comb Generation in a Silicon Nitride Chip, Opt. Lett. 36, 3398 (2011).

[22] Jiang Li, Hansuek Lee, Tong Chen, and Kerry J. Vahala, Low-Pump-Power, Low-Phase-Noise, and Microwave to Millimeter-Wave Repetition Rate Operation in Microcombs, Phys. Rev. Lett. 109, 233901 (2012).

[23] Anatoliy A. Savchenkov, Enrico Rubiola, Andrey B. Matsko, Vladimir S. Ilchenko, and Lute Maleki, Phase Noise of Whispering Gallery Photonic Hyper-Parametric Microwave Oscillators, Opt. Express 16, 4130 (2008).

[24] P. Del'Haye, O. Arcizet, A. Schliesser, R. Holzwarth, and T. J. Kippenberg, Full Stabilization of a MicroresonatorBased Optical Frequency Comb, Phys. Rev. Lett. 101, 053903 (2008). 
[25] T. Herr, K. Hartinger, J. Riemensberger, C. Y. Wang, E. Gavartin, R. L. Holzwarth, M. Gorodetsky, and T.J. Kippenberg, Universal Formation Dynamics and Noise of Kerr-Frequency Combs in Microresonators, Nat. Photonics 6, 480 (2012).

[26] M.L. Gorodetsky and I.S. Grudinin, Fundamental Thermal Fluctuations in Microspheres, J. Opt. Soc. Am. B 21, 697 (2004).

[27] Andrey B. Matsko, Anatoliy A. Savchenkov, Nan Yu, and Lute Maleki, Whispering-Gallery-Mode Resonators as Frequency References. I. Fundamental Limitations, J. Opt. Soc. Am. B 24, 1324 (2007).

[28] Pascal Del'Haye, Scott B. Papp, and Scott A. Diddams, Hybrid Electro-Optically Modulated Microcombs, Phys. Rev. Lett. 109, 263901 (2012).

[29] V.S Ilchenko, P. S Volikov, V. L Velichansky, F Treussart, V Lefevre-Seguin, J.-M Raimond, and S Haroche, StrainTunable High-Q Optical Microsphere Resonator, Opt. Commun. 145, 86 (1998).

[30] Wolf von Klitzing, Romain Long, Vladimir S Ilchenko, Jean Hare, and Valerie Lefevre-Seguin, Tunable
Whispering Gallery Modes for Spectroscopy and CQED Experiments, New J. Phys. 3, 14 (2001).

[31] Pump power can influence the line spacing quickly via the Kerr effect.

[32] See Supplemental Material at http://link.aps.org/ supplemental/10.1103/PhysRevX.3.031003 for details on $\mathrm{CO}_{2}$-laser machining, pump-laser and line-spacing stabilization.

[33] P. Del'Haye, S. A. Diddams, and S. B. Papp, Appl. Phys. Lett. 102, 221119 (2013).

[34] Ming Cai, Oskar Painter, and Kerry J. Vahala, Observation of Critical Coupling in a Fiber Taper to a Silica Microsphere Whispering-Gallery Mode System, Phys. Rev. Lett. 85, 74 (2000).

[35] Tal Carmon, Lan Yang, and Kerry Vahala, Dynamical Thermal Behavior and Thermal Self-Stability of Microcavities, Opt. Express 12, 4742 (2004).

[36] A. B. Matsko, A. A. Savchenkov, W. Liang, V. S. Ilchenko, D. Seidel, and L. Maleki, Optical Kerr Frequency Comb Generation in Overmoded Resonators, arXiv:1201.1959v1. 